\title{
Predictive Policing- The Future of Law Enforcement in the Trinidad and Tobago Police Service
}

\author{
Andre Norton \\ T \& T Police Service \\ Corner Edward \& Sackville Streets POS, Trinidad WI
}

\begin{abstract}
The Trinidad and Tobago Police Service (TTPS) is currently faced with large volumes of criminal data that continues to grow daily and which are required to be processed and transformed into useful information and where data mining can greatly improve crime analysis and aid in preventing and reducing crime. Currently, crime analysts attached to the analytical department of the TTPS are required to unravel the complexities in data to assist operational personnel in arresting offenders and also in directing crime prevention strategies. With the current volume of crime being committed and the awareness of modern criminals, this is becoming a very daunting task. The ability to analyze huge volumes of data with its inherent complexities without the use of computational support puts a strain on human resources. Because of the speed and advances in the field of data mining within recent years, independent studies on its impact on policing are only now getting on the way. It is particularly important in this respect to examine the benefits which the TTPS can derive through a careful implementation of this technology. The infamous events of July, 1990 in Trinidad and Tobago heralded the need for predictive policing and exacerbated concerns about national security by the local law enforcement agency. Accurately and efficiently analyzing the organization's ever growing volume of crime data is a major challenge facing the TTPS. This paper presents a case for implementing data mining (knowledge discovery in databases -KDD) within the TTPS as a tool for predictive analytics of crime data. It is hoped that this technology will provide decision makers with intelligence from the crime data to inform their strategic planning. It discusses the challenges of implementing data mining with special discussion of key issues relating to data integrity and the information technology (IT) infrastructure required to support data mining. It concludes by suggesting the internal information technology (IT) infrastructural changes needed to facilitate its implementation in the TTPS.
\end{abstract}

\section{General Terms}

Crime analysis, technological capabilities, law enforcement, fraud patterns, money laundering.

\section{Keywords}

Predictive policing, Data Mining, Artificial Intelligence, Uniform Crime Recording System (UCR)

\section{INTRODUCTION}

In Trinidad and Tobago, concerns about national security have increased significantly since the locally attempted terrorist acts of July $27^{\text {th }} 1990$. Most agencies charged with the responsibility for intelligence gathering have been actively collecting domestic intelligence to prevent any future attempts at insurrection. These efforts have significantly motivated the primary local law enforcement agency (the Trinidad and Tobago Police Service - TTPS) to more closely monitor criminal activities in all of its jurisdictions. Despite having adopted a computerized records management system and geographic information systems, the organization's ability to organize and assemble data about crime and disorder have not soared. In some aspects our technological capabilities have grown faster than our capacity to understand. The organization's ability to accurately and efficiently analyze the growing volumes of crime data poses a great challenge.

Trinidad and Tobago is a twin-island republic with an estimated population size of 1.3 million people [1] with a police agency (TTPS) comprising approximately 6,400 members [2]. In spite of in-house crime analysts, the organization is yet to explore data mining as a viable option for acquiring predictive capabilities from its vast storage of crime data.

Research has shown that data mining can greatly improve crime analysis and aid in reducing and preventing crime. As a matter of fact, no field is in greater need of data mining technology than law enforcement. One potential area of application is spatial data mining tools which provides law enforcement agencies with significant capabilities to learn crime trends on where, how and why crimes are committed.

An early application of data mining in law enforcement was by the FBI in the investigation of the Oklahoma City bombing case [3]. They adopted data mining techniques to scrutinize large volume of data gathered from various sources to track down criminals. Similarly, the Treasury Department of the United States adopted data mining technology to extract suspicious money laundering or fraud patterns [4]. This is particularly aiming at detecting criminals involved in money laundering or fraud.

The safety and security of citizens remains a major concern for the TTPS, however rather than focusing on enforcement and incarceration, the organization can deter crime through the knowledge benefits that derive from information and its associated technologies. By employing the correct technologies the organization can turn its officers into effective problem solvers by leveraging their intellectual capital to pre-empt crime. One such technology is data mining.

\section{KEY DEFINITIONS}

\subsection{What is Predictive Policing}

"Predictive policing is a concept that is built on the premise that it is possible to predict when and where crimes will occur 
again in the future by using sophisticated computer analysis of information about previously committed crimes [5]."

\subsection{What is Data Mining?}

In its simplest definition, data mining is the process of extracting patterns from data. It is the process of discovering interesting knowledge from large amounts of data stored either in databases, data warehouses or other information repositories. Crime data mining can be sub-divided into the following techniques:

- Clustering

- Classification

- Deviation Detection

- Social Network Analysis

- Entity Extraction

- Association Rule Mining

- String Comparator

- $\quad$ Sequential Pattern Mining

A single data mining tool or technique is not equally applicable to all the above-mentioned tasks. Based on the nature of the problem under consideration, and its proximity to the main divisions of the data mining techniques, choice of the appropriate technique is essential [6] .

\section{CRIME RECORDING PROCESS}

In the Trinidad and Tobago Police Service crime statistics is compiled using the Federal Bureau of Investigations (FBI) Uniform Recording System (UCR). UCR's successor, the National Incident Based Recording System (NIBRS) is yet to be implemented locally. There exist various specialist sections which investigate different crime types. For example all fraud matters are investigated by a specialized fraud unit; likewise stolen vehicles have a specialized investigative unit.

The ubiquitous nature of computing and the Internet's pervasiveness likewise makes offences such as identity theft, network intrusion, cyber piracy, and other illicit computermediated activities a new challenge for the TTPS.

Crime reports are documented on organizational specific general occurrence incident report (GOIR) forms which consists of structured fields (tick boxes) as well as an unstructured free form text area. Some of the key variables found on GOIR forms are day, date and time of crime (temporal dimensions), location of crime (spatial dimensions), offence type, victim and suspect data as well as modus operandi (MO) which identifies how the crime was committed. MO is documented in the unstructured free form text area of the crime form. There are approximately 400 uniform UCR codes. The contents of each form are manually entered into a centralized records management system (RMS) by data entry clerks.

\section{CHALLENGES TO DATA MINING IN TTPS}

At present, the greatest impediment to implementing data mining as a predictive policing tool within the TTPS is because of the following reasons:

- A large percentage of the data in the database is incomplete and invalid

- The data contain fields that requires parsing (elementizing) by being split into smaller parts before being able to use data mining tools on the database
- The data contain several abbreviations which should be changed to ensure consistency throughout the database (data standardization is needed)

- A significant portion of the data is incorrect

- There exists redundancy in the data (duplicate data stored at different locations in the database)

- The database have huge amounts of free form text that needs to be indexed and classified to be useful for data mining

- The data does not suitably enough match the business rules of the organization and there exist missing values, inconsistent values and invalid relationships

- There are several different forms of data that need to be converted to a single form of consistency in the database

In addition to these shortcomings, the database which supplies the raw data is dynamic (subject to update, append, delete etc.), which by default poses problems. Other unique problems arise as a consequence of these inadequacies and irrelevance of some of the stored information. This existing database like most databases was not designed to support data mining and as such, those essential attributes for knowledge discovery of the application domain are absent from the data, making it very difficult to discover significant knowledge about the given domain.

The quality of the data set is dependent upon a number of issues, but the source of the data is the crucial factor. Data entry and acquisition is inherently prone to errors both simple and complex. Much effort can be given to this front-end process, with respect to reduction in entry error, but the fact often remains that errors in a large data set are common [7]. Unless the organization takes extreme measures in an effort to avoid data errors the field errors rates will typically continue.

The current system for cleaning the data is more of a manual process which is laborious, time consuming and itself prone to errors. Analyzing poor quality data produces very little intelligence for the organization when designing its operational strategies for manpower deployment. One logical solution for improving data integrity is by incorporating it as part of the organization's business practices thereby reducing the need to consistently clean noisy data [8]. However, this pre-processing of data as a routine task usually consumes much of the efforts exerted in the entire data mining process. Therefore the potential to increase the usefulness of data by combining it with other data sources is great, but if the underlying data is not accurate, any relationships found in the data may be misleading.

A significant amount of disparately held databases exist among individual departments which require consolidation. Only the operational capabilities of geographical information systems (GIS) are being utilized. However where the administrative and strategic capabilities of the software is in greatest demand, the necessary skill set required for such utilization is in shortage across the wider organization.

\section{DATA MINING TECHNIQUES FOR CRIME PREDICTION}

Newer data mining techniques when applied to law enforcement identifies patterns form both structured and unstructured data. Similar to other forms of data mining that exist in the for profit world, there are privacy issues 
associated with crime data mining. However researchers and developers have created several automated data mining techniques specifically suited to police agencies [9]. The following are common crime data mining techniques designed for law enforcement:

- Clustering does not have a set of pre-defined classes for assigning items. They group data into classes with similar characteristics in order to maximize or minimize intra-class similarity. This could involve identifying suspects who conduct crimes in similar ways or distinguish among groups belonging to different gangs. Sometimes a statistic based concept space algorithm is used which automatically associate different objects e.g. persons, organizations and vehicles in crime records. Clustering crime incidents has a high computational intensity but automates a major part of crime analysis.

- Classification has been used to identify sources of e-mail spamming based upon the sender's structural features and linguistic patterns. Classification looks for common features between crime entities and organizes them into predefined classes. It is often used to predict crime trends and reduce the time required to identify crime entities but requires a predefined classification scheme. It also requires reasonably complete training and testing data because a high degree of missing data would limit prediction accuracy.

- Deviation detection is also referred to as outlier detection, uses specific measures to study data that differs markedly from the rest of the data. This technique is commonly applied to fraud detection, network intrusion detection, and other crime analyses, though in some instances these anomalies may be normal and thereby adding a further layer of complexity making it difficult to identify outliers.

- Social network analysis describes the roles of and interactions among nodes in a conceptual network. This technique can be used by investigators to build a network that illustrates criminals' roles, the flow of intangible and tangible information and entity associations. In spite of this technique enables investigators to visualize criminal networks, the discovery of the network's true leaders may be undiscoverable especially if such leaders are low profiled.

- Entity extraction identifies particular patterns from data such as text, images, or audio materials. It has been used to automatically identify persons, addresses, vehicles, and personal characteristics from police narrative reports. Entity extraction provides basic information for crime analysis, but its performance depends greatly on the availability of extensive amounts of clean input data.

- Association rule mining discovers frequently occurring item sets in a database and presents the patterns as rules. This technique has been applied in network intrusion detection to derive association rules from users' interaction history. Investigators also can apply this technique to network intruders' profiles to help detect potential future network attacks.

- String comparator techniques perform a comparison between textual fields in pairs of database records and compute the similarity between the records. These techniques can detect deceptive information such as name, address, and Identification Number in criminal records. Investigators can use string comparators to analyze textual data, but the techniques often require intensive computation.

- Sequential pattern mining is similar to association rule mining and this technique finds frequently occurring sequences of items over a set of transactions that occurred at different times. This approach identifies intrusion patterns in network intrusion detection among time-stamped data. Showing hidden patterns benefits crime analysis, but to obtain meaningful results requires rich and highly structured data.

\subsection{Phases of Data Mining}

- Data preparation phase- In this phase, the main data sets to be used by the data mining operation are identified and cleansed from any data impurities (data cleaning). If the data is in a data warehouse it will be already integrated and filtered, if not then it must be extracted from the operational database and cleaned before utilizing the data mining tools on the newly cleaned data.

- The data analysis and classification phase- The objective of this phase is to study the data in order to identify common data characteristics or patterns. The data mining tools applies specific algorithms to find the following:

- Data groupings, classifications, clusters, or sequences.

- Data dependencies, links, or relationships.

- Data patterns, trends, and deviations.

- The knowledge acquisition phase- This phase uses the results of the data analysis and classification phase. End user intervention selects the appropriate modeling or knowledge acquisition algorithms. Typically the algorithms used in data mining are based on neural networks, decision trees, rules induction, genetic algorithms, classification and regression trees, memory-based reasoning, or nearest neighbor and data visualization. Usually a data mining tool may use multiple algorithms in any combination to generate a computer model that reflects the behavior of the target data set.

- The prognosis phase- In this phase, the data mining findings are used to predict future behavior and forecast potential organizational outcomes. Examples of data mining findings can be as follows:

- $80 \%$ of Burglaries are committed by individuals who journeyed to commit the 
crime at a location distant from their homes. $55 \%$ of street Robberies was committed by persons of mixed descent between the ages of 21-25 yrs. from single parent households. $75 \%$ of stolen vehicles recovered were intact. The complete set of findings can be represented in a decision tree, a neural network, a forecasting model or a visual presentation interface which is then used to project future events or results. For example the prognosis phase may project the likely outcome of a new crime plan roll-out.

By uncovering critical patterns and variables that can be indicators of future crime activity, the TTPS can proactively recognize and react to threats to public safety before they take place [10].

\section{A CASE FOR DATA MINING}

Data mining is one of the fastest growing fields in the computer industry. Once a small interest area within computer science and statistics, it has quickly expanded into a field of its own. At present, most of the decision making process for operational deployment to crime prone areas is not supported by superior tools and techniques which can provide actionable intelligence from the stored crime data. Consequently, resource deployment, crime prevention and investigation strategies are being pursued more on the basis of crime incidents rather than crime patterns and trends.

This is a clear indication that the current approach toward crime prevention takes more a reactive than proactive approach. Data Mining will facilitate much more granularity for analyzing trends and pattern, which when combined with criminological theories can potentially provide the desired predictive capabilities to the organization. The advanced algorithms used by data mining technologies can allow the TTPS to better predict locations where crimes are likely to occur and direct appropriate resources to those areas. This effectively stops crimes before they occur.

Despite the extensive use of the TTPS' criminal database (RMS) by the agency's crime analysts to provide analytical products for decision making, the absence of an information technology infrastructure which supports analytical processing has thus far short-changed the organization in realizing its predictive policing capability (a proactive policing methodology) which is much need.

Currently, much emphasis is placed on counting crime and incidents. The crime prediction capabilities achievable from data mining presents the organization with opportunities to move from merely counting crimes more toward anticipating, preventing and perhaps responding more effectively to their occurrence. This focuses the organization more on effective use of its stored data to develop deployment strategies because criminal behavior tends to be relatively predictable which can be determined through analyzing historical data. Greater predictive capability is achievable utilizing data mining tools to exploit existing data sets in order to provide more actionable intelligence from the stored crime data.

\section{CONCLUSION}

Predictive policing is slowing making way to the forefront of strategic law enforcement and many policing experts believe

that it may be a prominent direction in the future. It involves anticipating questions which leads to a search for evidence, evidence helps officers establish facts and facts in turn support and substantiate action [11].

Many experts believe that predictive policing is the future of law enforcement. Predictive policing techniques when supported by the right tools can empower the TTPS to proactively fight crime by facilitating better forecasts of future incidents and events through the fast, accurate detention of patterns and trends from its large volumes of historical data residing in the RMS [12].

With the advanced predictive modeling solutions found in data mining, the organization can have the tools required to be in front of crime and stop criminals in their tracks, operate more cost effectively and boots public trust and confidence in the organization [13].

A combining of data mining techniques with dashboards, scorecards, data visualization, interactive mapping, geospatial analysis and enterprise search, the TTPS can overcome budgetary challenges and combat crime more successfully than before.

In order to achieve the aforementioned benefits it is imperative for the TTPS re-engineer the following:

- Data Collection- There is need to improve the current data collection methodology where data integrity becomes built-in to the internal business processes of the organization;

- IT Infrastructure upgrade- TTPS needs to upgrade the information technology infrastructure to facilitate predictive analytics technologies;

- Data Structure- All transactional criminal must be extracted from its operational environment, transformed into a data structure that facilitates analytical processing and stored electronically in a preferably non-volatile storage environment (e.g. a data warehouse);

- Data Integration- All disparate databases housing criminal and intelligence data must be integrated into this common data structure [14]. Other essential attributes from Court and Process data, CID/CRO data, prison release data and GIS data are required to complete this common structure.

It is important for police officers and crime analysts within TTPS to move the science forward, making predictions and acting on the results [15]. The goal of the organization should always be to reduce crime and improve service. Implementing data mining technologies as a predictive policing tool makes TTPS the pioneer ("first mover") police agency among Caricom member states to adopt the technique, thereby setting the stage for others to follow.

\section{ACKNOWLEDGMENTS}

I would like to pay special tribute to Dr. Christopher Ward, my supervisor at the University of the West Indies St. 
Augustine Campus Trinidad WI, who inspired me to write this paper.

\section{REFERENCES}

[1] Central statistical office 2012. Ministry of Planning and Development, Government of the Republic of Trinidad and Tobago. http://www.cso.gov.tt

[2] Human Resource Branch of the Trinidad and Tobago Police Service (HRB-TTPS)

[3] The Federal Bureau of Investigation, Terror Hits Home: The Oklahoma City Bombing

http://www.fbi.gov/about-us/history/famouscases/oklahoma-city-bombing

[4] Federal Agency Data Mining Report 2010, Department of Treasury January 2011

http://www.treasury.gov

[5] Uchida, C. 2009. National Institute of Justice: A National Discussion on Predictive Policing: Defining our Terms and Mapping Successful Implementation Strategies NJC 230404

[6] Adderley, R \& P.B. Musgrove 2001. Data Mining Case Study: Modeling the Behavior of Offenders Who Commit Serious Sexual Assaults, Proceedings of the seventh ACM SIGKDD international conference on Knowledge discovery and data mining.

[7] Brown, D., 2003.The Regional Crime Analysis Program (RECAP): A Framework for Mining Data to Catch
Criminals

http://vijis.sys.virginia.edu/publication/RECAP.pdf.

[8] Butler, A. 1992. Police Management $2^{\text {nd }}$ Edition, England: Dartmouth Publishing Company Limited.

[9] Franklin, D. 2002. Data Miners: New software instantly connects key bits of data that once eluded teams of researchers. Time, December 23.

[10] Helberg, C. 2002. Data mining with confidence, $2^{\text {nd }}$ Edition, SPSS, Inc., Chicago, IL.

[11] Mena, J. 2003. Investigative Data Mining for Security and Criminal Detection, Elsevier Science (USA).

[12] Mc Cue, C. et al, 2003. Data Mining and value-added analysis, FBI Law Enforcement Bulletin http://findarticles.com/p/articles/mi_m2194/is_11_72/ai_ 111496582

[13] Mc Cue, C. 2007. Data Mining and Predictive Analysis: Intelligence gathering and Crime Analysis, ButterworthHeinemann

[14] Perez, B. 2001. Data Mining Technology Use Grows; http://robotics.stanford.edu/users/ronnyk/kohavilnSCMP. pdf

[15] Tabussum, Z. 2003. CIA turns to data mining; http://www.parallaxresearch.com/news/2001/0309/cia_tu rns_to.html 